\title{
Inverse Trigonometric Functions Arctan and Arccot
}

\author{
Xiquan Liang \\ Qingdao University of Science \\ and Technology \\ China
}

\author{
Bing Xie \\ Qingdao University of Science \\ and Technology \\ China
}

Summary. This article describes definitions of inverse trigonometric functions arctan, arccot and their main properties, as well as several differentiation formulas of arctan and arccot.

MML identifier: SIN_COS9, version: $\underline{7.8 .10 \quad 4.100 .1011}$

The articles [17], [1], [2], [18], [3], [13], [19], [7], [15], [5], [9], [12], [16], [4], [6], [8], [11], [14], and [10] provide the notation and terminology for this paper.

\section{Function Arctan and Arccot}

For simplicity, we adopt the following convention: $x, r, s, h$ denote real numbers, $n$ denotes an element of $\mathbb{N}, Z$ denotes an open subset of $\mathbb{R}$, and $f, f_{1}$, $f_{2}$ denote partial functions from $\mathbb{R}$ to $\mathbb{R}$.

The following propositions are true:

(1) $]-\frac{\pi}{2}, \frac{\pi}{2}[\subseteq \operatorname{dom}$ (the function tan).

(2) $] 0, \pi[\subseteq \operatorname{dom}($ the function cot).

(3)(i) The function tan is differentiable on $]-\frac{\pi}{2}, \frac{\pi}{2}[$, and

(ii) for every $x$ such that $x \in]-\frac{\pi}{2}, \frac{\pi}{2}[\text { holds (the function } \tan )^{\prime}(x)=\frac{1}{(\cos x)^{2}}$.

(4) The function cot is differentiable on $] 0, \pi[$ and for every $x$ such that $x \in] 0, \pi\left[\right.$ holds $(\text { the function } \cot )^{\prime}(x)=-\frac{1}{(\sin x)^{2}}$.

(5) The function $\tan$ is continuous on $]-\frac{\pi}{2}, \frac{\pi}{2}[$.

(6) The function cot is continuous on $] 0, \pi[$. 
(7) The function tan is increasing on $]-\frac{\pi}{2}, \frac{\pi}{2}[$.

(8) The function cot is decreasing on $] 0, \pi[$.

(9) (The function $\tan ) \Gamma]-\frac{\pi}{2}, \frac{\pi}{2}[$ is one-to-one.

(10) (The function cot) $\uparrow] 0, \pi[$ is one-to-one.

Let us mention that (the function tan) $\rceil]-\frac{\pi}{2}, \frac{\pi}{2}$ [ is one-to-one and (the function cot) $\uparrow] 0, \pi[$ is one-to-one.

The partial function the function arctan from $\mathbb{R}$ to $\mathbb{R}$ is defined as follows:

(Def. 1) The function arctan $=\left((\right.$ the function $\tan )\lceil]-\frac{\pi}{2}, \frac{\pi}{2}[)^{-1}$.

The partial function the function arccot from $\mathbb{R}$ to $\mathbb{R}$ is defined by:

(Def. 2) The function arccot $=(($ the function cot $) \uparrow] 0, \pi[)^{-1}$.

Let $r$ be a real number. The functor $\arctan r$ is defined by:

(Def. 3) $\arctan r=($ the function $\arctan )(r)$.

The functor $\operatorname{arccot} r$ is defined by:

(Def. 4) $\operatorname{arccot} r=$ (the function $\operatorname{arccot})(r)$.

Let $r$ be a real number. Then $\arctan r$ is a real number. Then $\operatorname{arccot} r$ is a real number.

We now state two propositions:

(11) $\quad \mathrm{rng}$ (the function $\arctan )=]-\frac{\pi}{2}, \frac{\pi}{2}[$.

(12) $\mathrm{rng}($ the function arccot $)=] 0, \pi[$.

Let us mention that the function arctan is one-to-one and the function arccot is one-to-one.

Let $r$ be a real number. Then $\tan r$ is a real number. Then $\cot r$ is a real number.

Next we state a number of propositions:

(13) For every real number $x$ such that $x \in]-\frac{\pi}{2}, \frac{\pi}{2}[$ holds (the function $\tan )(x)=\tan x$.

(14) For every real number $x$ such that $x \in] 0, \pi[$ holds $($ the function $\cot )(x)=$ $\cot x$.

(15) For every real number $x$ such that $\cos x \neq 0$ holds (the function tan $)(x)=$ $\tan x$.

(16) For every real number $x$ such that (the function $\sin )(x) \neq 0$ holds (the function $\cot )(x)=\cot x$.

(17) $\tan \left(-\frac{\pi}{4}\right)=-1$.

(18) $\cot \left(\frac{\pi}{4}\right)=1$ and $\cot \left(\frac{3}{4} \cdot \pi\right)=-1$.

(19) For every real number $x$ such that $x \in\left[-\frac{\pi}{4}, \frac{\pi}{4}\right]$ holds $\tan x \in[-1,1]$.

(20) For every real number $x$ such that $x \in\left[\frac{\pi}{4}, \frac{3}{4} \cdot \pi\right]$ holds $\cot x \in[-1,1]$.

(21) $\operatorname{rng}\left((\right.$ the function $\left.\tan ) \uparrow\left[-\frac{\pi}{4}, \frac{\pi}{4}\right]\right)=[-1,1]$.

(22) $\operatorname{rng}\left((\right.$ the function cot $\left.) \uparrow\left[\frac{\pi}{4}, \frac{3}{4} \cdot \pi\right]\right)=[-1,1]$. 
(23) $[-1,1] \subseteq$ dom (the function arctan).

(24) $[-1,1] \subseteq \operatorname{dom}$ (the function arccot).

Let us observe that (the function $\tan )\left\lceil\left[-\frac{\pi}{4}, \frac{\pi}{4}\right]\right.$ is one-to-one and (the function cot) $\left\lceil\left[\frac{\pi}{4}, \frac{3}{4} \cdot \pi\right]\right.$ is one-to-one.

The following propositions are true:

(25) (The function arctan) $\uparrow[-1,1]=\left((\text { the function } \tan ) \uparrow\left[-\frac{\pi}{4}, \frac{\pi}{4}\right]\right)^{-1}$.

(26) (The function arccot) $\uparrow[-1,1]=\left((\text { the function cot }) \uparrow\left[\frac{\pi}{4}, \frac{3}{4} \cdot \pi\right]\right)^{-1}$.

(27) ((The function tan) $\uparrow\left[-\frac{\pi}{4}, \frac{\pi}{4}\right]$ qua function $) \cdot(($ the function arctan $) \uparrow[-1,1])=$ $\operatorname{id}_{[-1,1]}$.

(28) ((The function cot) $\uparrow\left[\frac{\pi}{4}, \frac{3}{4} \cdot \pi\right]$ qua function $) \cdot(($ the function arccot $) \uparrow[-1,1])=$ $\operatorname{id}_{[-1,1]}$.

(29) $\left((\right.$ The function $\tan )\left\lceil\left[-\frac{\pi}{4}, \frac{\pi}{4}\right]\right) \cdot(($ the function $\arctan ) \uparrow[-1,1])=\mathrm{id}_{[-1,1]}$.

(30) ((The function cot) $\left.\uparrow\left[\frac{\pi}{4}, \frac{3}{4} \cdot \pi\right]\right) \cdot(($ the function $\operatorname{arccot}) \uparrow[-1,1])=\operatorname{id}_{[-1,1]}$.

(31) (The function arctan qua function) $\cdot(($ the function $\tan ) \uparrow]-\frac{\pi}{2}, \frac{\pi}{2}[)=$ $\mathrm{id}_{]}-\frac{\pi}{2}, \frac{\pi}{2}[\cdot$

(32) (The function arccot) $\cdot\left((\right.$ the function cot) $)\left[0, \pi[)=\mathrm{id}_{] 0, \pi[}[\right.$.

(33) (The function arctan qua function) $\cdot($ (the function $\tan )\lceil]-\frac{\pi}{2}, \frac{\pi}{2}[)=$ $\mathrm{id}_{]-\frac{\pi}{2}, \frac{\pi}{2}[} \cdot$

(34) (The function arccot qua function) $\cdot\left((\right.$ the function cot) $\mid] 0, \pi[)=\mathrm{id}_{] 0, \pi[}$.

(35) If $-\frac{\pi}{2}<r<\frac{\pi}{2}$, then $\arctan \tan r=r$.

(36) If $0<r<\pi$, then $\operatorname{arccot} \cot r=r$.

(37) $\arctan (-1)=-\frac{\pi}{4}$.

(38) $\operatorname{arccot}(-1)=\frac{3}{4} \cdot \pi$.

(39) $\arctan 1=\frac{\pi}{4}$.

(40) $\operatorname{arccot} 1=\frac{\pi}{4}$.

(41) $\tan 0=0$.

(42) $\cot \left(\frac{\pi}{2}\right)=0$.

(43) $\arctan 0=0$.

(44) $\operatorname{arccot} 0=\frac{\pi}{2}$.

(45) The function arctan is increasing on (the function tan $\left.)^{\circ}\right]-\frac{\pi}{2}, \frac{\pi}{2}[$.

(46) The function arccot is decreasing on (the function cot) $\left.{ }^{\circ}\right] 0, \pi[$.

(47) The function arctan is increasing on $[-1,1]$.

(48) The function arccot is decreasing on $[-1,1]$.

(49) For every real number $x$ such that $x \in[-1,1]$ holds $\arctan x \in\left[-\frac{\pi}{4}, \frac{\pi}{4}\right]$.

(50) For every real number $x$ such that $x \in[-1,1]$ holds $\operatorname{arccot} x \in\left[\frac{\pi}{4}, \frac{3}{4} \cdot \pi\right]$.

(51) If $-1 \leq r \leq 1$, then $\tan \arctan r=r$.

(52) If $-1 \leq r \leq 1$, then $\cot \operatorname{arccot} r=r$. 
(53) The function arctan is continuous on $[-1,1]$.

(54) The function arccot is continuous on $[-1,1]$.

(55) $\operatorname{rng}(($ the function $\arctan ) \uparrow[-1,1])=\left[-\frac{\pi}{4}, \frac{\pi}{4}\right]$.

(56) $\operatorname{rng}\left((\right.$ the function arccot $)\lceil[-1,1])=\left[\frac{\pi}{4}, \frac{3}{4} \cdot \pi\right]$.

(57) If $-1 \leq r \leq 1$ and $\arctan r=-\frac{\pi}{4}$, then $r=-1$.

(58) If $-1 \leq r \leq 1$ and $\operatorname{arccot} r=\frac{3}{4} \cdot \pi$, then $r=-1$.

(59) If $-1 \leq r \leq 1$ and $\arctan r=0$, then $r=0$.

(60) If $-1 \leq r \leq 1$ and $\operatorname{arccot} r=\frac{\pi}{2}$, then $r=0$.

(61) If $-1 \leq r \leq 1$ and $\arctan r=\frac{\pi}{4}$, then $r=1$.

(62) If $-1 \leq r \leq 1$ and $\operatorname{arccot} r=\frac{\pi}{4}$, then $r=1$.

(63) If $-1 \leq r \leq 1$, then $-\frac{\pi}{4} \leq \arctan r \leq \frac{\pi}{4}$.

(64) If $-1 \leq r \leq 1$, then $\frac{\pi}{4} \leq \operatorname{arccot} r \leq \frac{3}{4} \cdot \pi$.

(65) If $-1<r<1$, then $-\frac{\pi}{4}<\arctan r<\frac{\pi}{4}$.

(66) If $-1<r<1$, then $\frac{\pi}{4}<\operatorname{arccot} r<\frac{3}{4} \cdot \pi$.

(67) If $-1 \leq r \leq 1$, then $\arctan r=-\arctan (-r)$.

(68) If $-1 \leq r \leq 1$, then $\operatorname{arccot} r=\pi-\operatorname{arccot}(-r)$.

(69) If $-1 \leq r \leq 1$, then $\cot \arctan r=\frac{1}{r}$.

(70) If $-1 \leq r \leq 1$, then $\tan \operatorname{arccot} r=\frac{1}{r}$.

(71) The function arctan is differentiable on (the function tan) $\left.{ }^{\circ}\right]-\frac{\pi}{2}, \frac{\pi}{2}[$.

(72) The function arccot is differentiable on (the function cot) $\left.{ }^{\circ}\right] 0, \pi[$.

(73) The function arctan is differentiable on $]-1,1[$.

(74) The function arccot is differentiable on $]-1,1[$.

(75) If $-1 \leq r \leq 1$, then (the function $\arctan ^{\prime}(r)=\frac{1}{1+r^{2}}$.

(76) If $-1 \leq r \leq 1$, then (the function $\operatorname{arccot})^{\prime}(r)=-\frac{1}{1+r^{2}}$.

(77) The function arctan is continuous on (the function tan) $\left.{ }^{\circ}\right]-\frac{\pi}{2}, \frac{\pi}{2}[$.

(78) The function arccot is continuous on (the function cot) $\left.{ }^{\circ}\right] 0, \pi[$.

(79) dom (the function arctan) is open.

(80) dom (the function arccot) is open.

\section{Several Differentiation Formulas of Arctan and Arccot}

We now state a number of propositions:

(81) Suppose $Z \subseteq]-1,1[$. Then the function $\arctan$ is differentiable on $Z$ and for every $x$ such that $x \in Z$ holds (the function $\arctan )^{\prime}{ }_{\mid Z}(x)=\frac{1}{1+x^{2}}$.

(82) Suppose $Z \subseteq]-1,1[$. Then the function arccot is differentiable on $Z$ and for every $x$ such that $x \in Z$ holds (the function $\operatorname{arccot})^{\prime}{ }_{\mid Z}(x)=-\frac{1}{1+x^{2}}$. 
(83) Suppose $Z \subseteq]-1,1[$. Then

(i) $\quad r$ the function arctan is differentiable on $Z$, and

(ii) for every $x$ such that $x \in Z$ holds $\left(r\right.$ the function $\arctan ^{\prime}{ }_{{ }^{Z}}(x)=\frac{r}{1+x^{2}}$.

(84) Suppose $Z \subseteq]-1,1[$. Then

(i) $\quad r$ the function arccot is differentiable on $Z$, and

(ii) for every $x$ such that $x \in Z$ holds $(r \text { the function } \operatorname{arccot})^{\prime}{ }_{Y Z}(x)=-\frac{r}{1+x^{2}}$.

(85) Suppose $f$ is differentiable in $x$ and $-1<f(x)<1$. Then (the function arctan $) \cdot f$ is differentiable in $x$ and $((\text { the function } \arctan ) \cdot f)^{\prime}(x)=$ $\frac{f^{\prime}(x)}{1+f(x)^{2}}$.

(86) Suppose $f$ is differentiable in $x$ and $-1<f(x)<1$. Then (the function arccot $) \cdot f$ is differentiable in $x$ and $((\text { the function arccot }) \cdot f)^{\prime}(x)=$ $-\frac{f^{\prime}(x)}{1+f(x)^{2}}$

(87) Suppose $Z \subseteq \operatorname{dom}(($ the function $\arctan ) \cdot f)$ and for every $x$ such that $x \in Z$ holds $f(x)=r \cdot x+s$ and $-1<f(x)<1$. Then

(i) (the function $\arctan ) \cdot f$ is differentiable on $Z$, and

(ii) for every $x$ such that $x \in Z$ holds ((the function $\arctan ) \cdot f)_{{ }^{\prime}}^{\prime}(x)=$ $\frac{r}{1+(r \cdot x+s)^{2}}$.

(88) Suppose $Z \subseteq \operatorname{dom}(($ the function $\operatorname{arccot}) \cdot f)$ and for every $x$ such that $x \in Z$ holds $f(x)=r \cdot x+s$ and $-1<f(x)<1$. Then

(i) (the function arccot) $\cdot f$ is differentiable on $Z$, and

(ii) for every $x$ such that $x \in Z$ holds ((the function $\operatorname{arccot)} \cdot f)^{\prime}{ }_{{ }_{Z}}(x)=$ $-\frac{r}{1+(r \cdot x+s)^{2}}$.

(89) Suppose $Z \subseteq \operatorname{dom}(($ the function $\ln ) \cdot($ the function arctan) $)$ and $Z \subseteq$ ] $-1,1$ [ and for every $x$ such that $x \in Z$ holds $\arctan x>0$. Then

(i) (the function $\ln$ ) (the function arctan) is differentiable on $Z$, and

(ii) for every $x$ such that $x \in Z$ holds ((the function $\ln$ ) ·(the function $\arctan ))_{\uparrow Z}^{\prime}(x)=\frac{1}{\left(1+x^{2}\right) \cdot \arctan x}$.

(90) Suppose $Z \subseteq \operatorname{dom}(($ the function $\ln ) \cdot($ the function arccot)) and $Z \subseteq$ ] $-1,1[$ and for every $x$ such that $x \in Z$ holds $\operatorname{arccot} x>0$. Then

(i) (the function $\ln$ ) $\cdot$ (the function arccot) is differentiable on $Z$, and

(ii) for every $x$ such that $x \in Z$ holds ((the function $\ln$ ) ·(the function $\operatorname{arccot}))_{i Z}^{\prime}(x)=-\frac{1}{\left(1+x^{2}\right) \cdot \operatorname{arccot} x}$.

(91) Suppose $Z \subseteq \operatorname{dom}\left(\left(\square^{n}\right) \cdot\right.$ the function $\left.\arctan \right)$ and $\left.Z \subseteq\right]-1,1[$. Then

(i) $\left(\square^{n}\right) \cdot$ the function arctan is differentiable on $Z$, and

(ii) for every $x$ such that $x \in Z$ holds $\left(\left(\square^{n}\right) \cdot \text { the function } \arctan \right)^{\prime}{ }_{\mid Z}(x)=$ $\frac{n \cdot(\arctan x)^{n-1}}{1+x^{2}}$.

(92) Suppose $Z \subseteq \operatorname{dom}\left(\left(\square^{n}\right) \cdot\right.$ the function arccot $)$ and $\left.Z \subseteq\right]-1,1[$. Then

(i) $\left(\square^{n}\right) \cdot$ the function arccot is differentiable on $Z$, and 
(ii) for every $x$ such that $x \in Z$ holds $\left(\left(\square^{n}\right) \cdot\right.$ the function $\operatorname{arccot}^{\prime}{ }_{\ulcorner Z}(x)=$ $-\frac{n \cdot(\operatorname{arccot} x)^{n-1}}{1+x^{2}}$.

(93) Suppose $Z \subseteq \operatorname{dom}\left(\frac{1}{2}\left(\left(\square^{2}\right) \cdot\right.\right.$ the function $\left.\left.\arctan \right)\right)$ and $\left.Z \subseteq\right]-1,1[$. Then

(i) $\quad \frac{1}{2}\left(\left(\square^{2}\right) \cdot\right.$ the function arctan) is differentiable on $Z$, and

(ii) for every $x$ such that $x \in Z$ holds $\left(\frac{1}{2}\left(\left(\square^{2}\right) \text {. the function } \arctan \right)\right)^{\prime}{ }_{\mid Z}(x)=$ $\frac{\arctan x}{1+x^{2}}$.

(94) Suppose $Z \subseteq \operatorname{dom}\left(\frac{1}{2}\left(\left(\square^{2}\right) \cdot\right.\right.$ the function arccot $\left.)\right)$ and $\left.Z \subseteq\right]-1,1[$. Then

(i) $\frac{1}{2}\left(\left(\square^{2}\right) \cdot\right.$ the function arccot) is differentiable on $Z$, and

(ii) for every $x$ such that $x \in Z$ holds $\left(\frac{1}{2}\left(\left(\square^{2}\right) \text {. the function } \operatorname{arccot}\right)\right)^{\prime}{ }_{Z}(x)=$ $-\frac{\operatorname{arccot} x}{1+x^{2}}$.

(95) Suppose $Z \subseteq]-1,1[$. Then

(i) $\operatorname{id}_{Z}$ the function $\arctan$ is differentiable on $Z$, and

(ii) for every $x$ such that $x \in Z$ holds $\left(\operatorname{id}_{Z} \text { the function } \arctan \right)_{{ }^{\prime}}^{\prime}(x)=$ $\arctan x+\frac{x}{1+x^{2}}$.

(96) Suppose $Z \subseteq]-1,1[$. Then

(i) $\operatorname{id}_{Z}$ the function arccot is differentiable on $Z$, and

(ii) for every $x$ such that $x \in Z$ holds $\left(\operatorname{id}_{Z}\right.$ the function $\operatorname{arccot}^{\prime}{ }_{i Z}(x)=$ $\operatorname{arccot} x-\frac{x}{1+x^{2}}$.

(97) Suppose $Z \subseteq \operatorname{dom}(f$ the function arctan) and $Z \subseteq]-1,1[$ and for every $x$ such that $x \in Z$ holds $f(x)=r \cdot x+s$. Then

(i) $f$ the function arctan is differentiable on $Z$, and

(ii) for every $x$ such that $x \in Z$ holds ( $f$ the function $\arctan )^{\prime}{ }_{{ }^{\prime}}(x)=$ $r \cdot \arctan x+\frac{r \cdot x+s}{1+x^{2}}$.

(98) Suppose $Z \subseteq \operatorname{dom}(f$ the function arccot) and $Z \subseteq]-1,1[$ and for every $x$ such that $x \in Z$ holds $f(x)=r \cdot x+s$. Then

(i) $f$ the function arccot is differentiable on $Z$, and

(ii) for every $x$ such that $x \in Z$ holds ( $f$ the function $\operatorname{arccot}^{\prime}{ }_{\mid Z}(x)=$ $r \cdot \operatorname{arccot} x-\frac{r \cdot x+s}{1+x^{2}}$.

(99) Suppose $Z \subseteq \operatorname{dom}\left(\frac{1}{2}((\right.$ the function $\left.\arctan ) \cdot f)\right)$ and for every $x$ such that $x \in Z$ holds $f(x)=2 \cdot x$ and $-1<f(x)<1$. Then

(i) $\frac{1}{2}(($ the function $\arctan ) \cdot f)$ is differentiable on $Z$, and

(ii) for every $x$ such that $x \in Z$ holds $\left(\frac{1}{2}((\text { the function } \arctan ) \cdot f)\right)^{\prime}{ }_{\mid Z}(x)=$ $\frac{1}{1+(2 \cdot x)^{2}}$.

(100) Suppose $Z \subseteq \operatorname{dom}\left(\frac{1}{2}((\right.$ the function $\left.\operatorname{arccot}) \cdot f)\right)$ and for every $x$ such that $x \in Z$ holds $f(x)=2 \cdot x$ and $-1<f(x)<1$. Then

(i) $\frac{1}{2}(($ the function arcot) $\cdot f)$ is differentiable on $Z$, and

(ii) for every $x$ such that $x \in Z$ holds $\left(\frac{1}{2}((\text { the function arccot }) \cdot f)\right)^{\prime}{ }_{Z}(x)=$ $-\frac{1}{1+(2 \cdot x)^{2}}$.

(101) Suppose $Z \subseteq \operatorname{dom}\left(f_{1}+f_{2}\right)$ and for every $x$ such that $x \in Z$ holds $f_{1}(x)=1$ and $f_{2}=\square^{2}$. Then $f_{1}+f_{2}$ is differentiable on $Z$ and for every 
$x$ such that $x \in Z$ holds $\left(f_{1}+f_{2}\right)^{\prime}{ }_{{ }_{Z}}(x)=2 \cdot x$.

(102) Suppose $Z \subseteq \operatorname{dom}\left(\frac{1}{2}\right.$ ((the function $\left.\left.\left.\ln \right) \cdot\left(f_{1}+f_{2}\right)\right)\right)$ and $f_{2}=\square^{2}$ and for every $x$ such that $x \in Z$ holds $f_{1}(x)=1$. Then

(i) $\frac{1}{2}\left((\right.$ the function $\left.\ln ) \cdot\left(f_{1}+f_{2}\right)\right)$ is differentiable on $Z$, and

(ii) for every $x$ such that $x \in Z$ holds $\left(\frac{1}{2}\right.$ ((the function $\left.\ln \right) \cdot\left(f_{1}+\right.$ $\left.\left.\left.f_{2}\right)\right)\right)_{\lceil Z}^{\prime}(x)=\frac{x}{1+x^{2}}$.

(103) Suppose that

(i) $Z \subseteq \operatorname{dom}\left(\operatorname{id}_{Z}\right.$ the function $\arctan -\frac{1}{2}\left((\right.$ the function $\left.\left.\ln ) \cdot\left(f_{1}+f_{2}\right)\right)\right)$,

(ii) $Z \subseteq]-1,1[$,

(iii) $f_{2}=\square^{2}$, and

(iv) for every $x$ such that $x \in Z$ holds $f_{1}(x)=1$.

Then

(v) $\operatorname{id}_{Z}$ the function $\arctan -\frac{1}{2}\left((\right.$ the function $\left.\ln ) \cdot\left(f_{1}+f_{2}\right)\right)$ is differentiable on $Z$, and

(vi) for every $x$ such that $x \in Z$ holds (id $Z$ the function $\arctan -\frac{1}{2}$ ((the function $\left.\left.\ln ) \cdot\left(f_{1}+f_{2}\right)\right)\right)_{\lceil Z}^{\prime}(x)=\arctan x$.

(104) Suppose that

(i) $Z \subseteq \operatorname{dom}\left(\operatorname{id}_{Z}\right.$ the function $\operatorname{arccot}+\frac{1}{2}\left((\right.$ the function $\left.\left.\ln ) \cdot\left(f_{1}+f_{2}\right)\right)\right)$,

(ii) $Z \subseteq]-1,1[$,

(iii) $f_{2}=\square^{2}$, and

(iv) for every $x$ such that $x \in Z$ holds $f_{1}(x)=1$.

Then

(v) $\operatorname{id}_{Z}$ the function $\operatorname{arccot}+\frac{1}{2}\left((\right.$ the function $\left.\ln ) \cdot\left(f_{1}+f_{2}\right)\right)$ is differentiable on $Z$, and

(vi) for every $x$ such that $x \in Z$ holds (id $Z$ the function $\operatorname{arccot}+\frac{1}{2}$ ((the function $\left.\left.\ln ) \cdot\left(f_{1}+f_{2}\right)\right)\right)^{\prime}{ }_{Z Z}^{\prime}(x)=\operatorname{arccot} x$.

(105) Suppose $Z \subseteq \operatorname{dom}\left(\operatorname{id}_{Z}((\right.$ the function $\left.\arctan ) \cdot f)\right)$ and for every $x$ such that $x \in Z$ holds $f(x)=\frac{x}{r}$ and $-1<f(x)<1$. Then

(i) $\operatorname{id}_{Z}(($ the function $\arctan ) \cdot f)$ is differentiable on $Z$, and

(ii) for every $x$ such that $x \in Z$ holds (id $Z$ ((the function arctan) $\cdot f))_{\lceil Z}^{\prime}(x)=\arctan \left(\frac{x}{r}\right)+\frac{x}{r \cdot\left(1+\left(\frac{x}{r}\right)^{2}\right)}$.

(106) Suppose $Z \subseteq \operatorname{dom}\left(\operatorname{id}_{Z}((\right.$ the function arccot $\left.) \cdot f)\right)$ and for every $x$ such that $x \in Z$ holds $f(x)=\frac{x}{r}$ and $-1<f(x)<1$. Then

(i) $\operatorname{id}_{Z}(($ the function arccot $) \cdot f)$ is differentiable on $Z$, and

(ii) for every $x$ such that $x \in Z$ holds $\left(\operatorname{id}_{Z}((\text { the function } \operatorname{arccot}) \cdot f)\right)^{\prime}{ }_{Z}(x)=$ $\operatorname{arccot}\left(\frac{x}{r}\right)-\frac{x}{r \cdot\left(1+\left(\frac{x}{r}\right)^{2}\right)}$

(107) Suppose $Z \subseteq \operatorname{dom}\left(f_{1}+f_{2}\right)$ and for every $x$ such that $x \in Z$ holds $f_{1}(x)=1$ and $f_{2}=\left(\square^{2}\right) \cdot f$ and for every $x$ such that $x \in Z$ holds $f(x)=\frac{x}{r}$. Then $f_{1}+f_{2}$ is differentiable on $Z$ and for every $x$ such that $x \in Z$ holds $\left(f_{1}+f_{2}\right)_{\lceil Z}^{\prime}(x)=\frac{2 \cdot x}{r^{2}}$. 
(108) Suppose that

(i) $Z \subseteq \operatorname{dom}\left(\frac{r}{2}\left((\right.\right.$ the function $\left.\left.\ln ) \cdot\left(f_{1}+f_{2}\right)\right)\right)$,

(ii) for every $x$ such that $x \in Z$ holds $f_{1}(x)=1$,

(iii) $r \neq 0$,

(iv) $f_{2}=\left(\square^{2}\right) \cdot f$, and

(v) for every $x$ such that $x \in Z$ holds $f(x)=\frac{x}{r}$.

Then

(vi) $\frac{r}{2}\left((\right.$ the function $\left.\ln ) \cdot\left(f_{1}+f_{2}\right)\right)$ is differentiable on $Z$, and

(vii) for every $x$ such that $x \in Z$ holds $\left(\frac{r}{2}\right.$ ((the function $\left.\ln \right) \cdot\left(f_{1}+\right.$ $\left.\left.\left.f_{2}\right)\right)\right)_{{ }^{\prime} Z}^{\prime}(x)=\frac{x}{r \cdot\left(1+\left(\frac{x}{r}\right)^{2}\right)}$.

(109) Suppose that

(i) $Z \subseteq \operatorname{dom}\left(\operatorname{id}_{Z}((\right.$ the function $\arctan ) \cdot f)-\frac{r}{2}\left((\right.$ the function $\left.\left.\ln ) \cdot\left(f_{1}+f_{2}\right)\right)\right)$,

(ii) $r \neq 0$,

(iii) for every $x$ such that $x \in Z$ holds $f(x)=\frac{x}{r}$ and $-1<f(x)<1$,

(iv) for every $x$ such that $x \in Z$ holds $f_{1}(x)=1$,

(v) $f_{2}=\left(\square^{2}\right) \cdot f$, and

(vi) for every $x$ such that $x \in Z$ holds $f(x)=\frac{x}{r}$.

Then

(vii) $\operatorname{id}_{Z}(($ the function $\arctan ) \cdot f)-\frac{r}{2}\left((\right.$ the function $\left.\ln ) \cdot\left(f_{1}+f_{2}\right)\right)$ is differentiable on $Z$, and

(viii) for every $x$ such that $x \in Z$ holds $\left(\operatorname{id}_{Z}((\right.$ the function $\arctan ) \cdot f)-$ $\frac{r}{2}\left((\right.$ the function $\left.\left.\ln ) \cdot\left(f_{1}+f_{2}\right)\right)\right)^{\prime}{ }_{Z}(x)=\arctan \left(\frac{x}{r}\right)$.

(110) Suppose that

(i) $Z \subseteq \operatorname{dom}\left(\operatorname{id}_{Z}((\right.$ the function $\operatorname{arccot}) \cdot f)+\frac{r}{2}\left((\right.$ the function $\left.\left.\ln ) \cdot\left(f_{1}+f_{2}\right)\right)\right)$,

(ii) $r \neq 0$,

(iii) for every $x$ such that $x \in Z$ holds $f(x)=\frac{x}{r}$ and $-1<f(x)<1$,

(iv) for every $x$ such that $x \in Z$ holds $f_{1}(x)=1$,

(v) $f_{2}=\left(\square^{2}\right) \cdot f$, and

(vi) for every $x$ such that $x \in Z$ holds $f(x)=\frac{x}{r}$.

Then

(vii) $\quad \operatorname{id}_{Z}(($ the function arccot $) \cdot f)+\frac{r}{2}\left((\right.$ the function $\left.\ln ) \cdot\left(f_{1}+f_{2}\right)\right)$ is differentiable on $Z$, and

(viii) for every $x$ such that $x \in Z$ holds $\left(\operatorname{id}_{Z}((\right.$ the function arccot $) \cdot f)+\frac{r}{2}(($ the function $\left.\left.\ln ) \cdot\left(f_{1}+f_{2}\right)\right)\right)_{{ }^{\prime}}^{\prime}(x)=\operatorname{arccot}\left(\frac{x}{r}\right)$.

(111) Suppose $Z \subseteq \operatorname{dom}\left((\right.$ the function $\left.\arctan ) \cdot \frac{1}{f}\right)$ and for every $x$ such that $x \in Z$ holds $f(x)=x$ and $-1<\left(\frac{1}{f}\right)(x)<1$. Then

(i) (the function $\arctan ) \cdot \frac{1}{f}$ is differentiable on $Z$, and

(ii) for every $x$ such that $x \in Z$ holds ((the function $\left.\arctan ) \cdot \frac{1}{f}\right)_{\mid Z}^{\prime}(x)=$ $-\frac{1}{1+x^{2}}$. 
(112) Suppose $Z \subseteq \operatorname{dom}\left(\left(\right.\right.$ the function arccot) $\left.\cdot \frac{1}{f}\right)$ and for every $x$ such that $x \in Z$ holds $f(x)=x$ and $-1<\left(\frac{1}{f}\right)(x)<1$. Then

(i) (the function arccot) $\cdot \frac{1}{f}$ is differentiable on $Z$, and

(ii) for every $x$ such that $x \in Z$ holds ((the function $\left.\operatorname{arccot)} \cdot \frac{1}{f}\right)_{{ }^{\prime}}^{\prime}(x)=$ $\frac{1}{1+x^{2}}$.

(113) Suppose that

(i) $Z \subseteq \operatorname{dom}(($ the function $\arctan ) \cdot f)$,

(ii) $f=f_{1}+h f_{2}$,

(iii) for every $x$ such that $x \in Z$ holds $-1<f(x)<1$,

(iv) for every $x$ such that $x \in Z$ holds $f_{1}(x)=r+s \cdot x$, and

(v) $f_{2}=\square^{2}$.

Then

(vi) (the function arctan) $\cdot\left(f_{1}+h f_{2}\right)$ is differentiable on $Z$, and

(vii) for every $x$ such that $x \in Z$ holds ((the function arctan) $\cdot\left(f_{1}+\right.$ $\left.\left.h f_{2}\right)\right)_{\lceil Z}^{\prime}(x)=\frac{s+2 \cdot h \cdot x}{1+\left(r+s \cdot x+h \cdot x^{2}\right)^{2}}$.

(114) Suppose that

(i) $Z \subseteq \operatorname{dom}(($ the function $\operatorname{arccot}) \cdot f)$,

(ii) $f=f_{1}+h f_{2}$,

(iii) for every $x$ such that $x \in Z$ holds $-1<f(x)<1$,

(iv) for every $x$ such that $x \in Z$ holds $f_{1}(x)=r+s \cdot x$, and

(v) $f_{2}=\square^{2}$.

Then

(vi) (the function arccot) $\cdot\left(f_{1}+h f_{2}\right)$ is differentiable on $Z$, and

(vii) for every $x$ such that $x \in Z$ holds ((the function arccot) $\cdot\left(f_{1}+\right.$ $\left.\left.h f_{2}\right)\right)_{{ }^{\prime} Z}^{\prime}(x)=-\frac{s+2 \cdot h \cdot x}{1+\left(r+s \cdot x+h \cdot x^{2}\right)^{2}}$.

(115) Suppose $Z \subseteq \operatorname{dom}(($ the function $\arctan ) \cdot($ the function exp)) and for every $x$ such that $x \in Z$ holds $\exp x<1$. Then

(i) (the function arctan) (the function exp) is differentiable on $Z$, and

(ii) for every $x$ such that $x \in Z$ holds ((the function arctan) $\cdot$ (the function $\exp ))_{\lceil Z}^{\prime}(x)=\frac{\exp x}{1+(\exp x)^{2}}$

(116) Suppose $Z \subseteq \operatorname{dom}(($ the function arccot) $\cdot($ the function exp)) and for every $x$ such that $x \in Z$ holds $\exp x<1$. Then

(i) (the function arccot) (the function exp) is differentiable on $Z$, and

(ii) for every $x$ such that $x \in Z$ holds ((the function arccot) $\cdot$ (the function $\exp ))_{{ }^{\prime}}^{\prime}(x)=-\frac{\exp x}{1+(\exp x)^{2}}$.

(117) Suppose that

(i) $Z \subseteq \operatorname{dom}(($ the function $\arctan ) \cdot($ the function $\ln ))$, and

(ii) for every $x$ such that $x \in Z$ holds $-1<$ (the function $\ln$ ) $(x)$ and (the function $\ln )(x)<1$.

Then 
(iii) (the function arctan) (the function $\ln$ ) is differentiable on $Z$, and

(iv) for every $x$ such that $x \in Z$ holds ((the function arctan) $\cdot$ (the function $\ln ))^{\prime}{ }_{Z}(x)=\frac{1}{x \cdot\left(1+(\text { the function } \ln )(x)^{2}\right)}$.

(118) Suppose that

(i) $Z \subseteq \operatorname{dom}(($ the function arccot) $\cdot($ the function $\ln ))$, and

(ii) for every $x$ such that $x \in Z$ holds $-1<$ (the function $\ln )(x)$ and (the function $\ln )(x)<1$.

Then

(iii) (the function arccot) (the function ln) is differentiable on $Z$, and

(iv) for every $x$ such that $x \in Z$ holds ((the function arccot) $\cdot$ (the function $\ln ))_{\uparrow Z}^{\prime}(x)=-\frac{1}{x \cdot\left(1+(\text { the function } \ln )(x)^{2}\right)}$.

(119) Suppose $Z \subseteq \operatorname{dom}(($ the function exp) $\cdot($ the function arctan $))$ and $Z \subseteq$ ]$-1,1[$. Then

(i) (the function exp) ·(the function arctan) is differentiable on $Z$, and

(ii) for every $x$ such that $x \in Z$ holds ((the function exp) $\cdot($ the function $\arctan ))_{\uparrow Z}^{\prime}(x)=\frac{\exp \arctan x}{1+x^{2}}$

(120) Suppose $Z \subseteq \operatorname{dom}(($ the function $\exp ) \cdot($ the function arccot $))$ and $Z \subseteq$ ]$-1,1[$. Then

(i) (the function exp) (the function arccot) is differentiable on $Z$, and

(ii) for every $x$ such that $x \in Z$ holds ((the function exp) ·(the function $\operatorname{arccot}))_{i Z}^{\prime}(x)=-\frac{\exp \operatorname{arccot} x}{1+x^{2}}$.

(121) Suppose $Z \subseteq \operatorname{dom}\left((\right.$ the function $\left.\arctan )-\operatorname{id}_{Z}\right)$ and $\left.Z \subseteq\right]-1,1[$. Then

(i) (the function $\arctan )-\mathrm{id}_{Z}$ is differentiable on $Z$, and

(ii) for every $x$ such that $x \in Z$ holds ((the function $\left.\arctan )-\mathrm{id}_{Z}\right)^{\prime}{ }_{Z}(x)=$ $-\frac{x^{2}}{1+x^{2}}$.

(122) Suppose $Z \subseteq \operatorname{dom}\left(-\right.$ the function $\left.\operatorname{arccot}-\operatorname{id}_{Z}\right)$ and $\left.Z \subseteq\right]-1,1[$. Then

(i) -the function $\operatorname{arccot}-\mathrm{id}_{Z}$ is differentiable on $Z$, and

(ii) for every $x$ such that $x \in Z$ holds (-the function $\left.\operatorname{arccot}-\operatorname{id}_{Z}\right)_{{ }^{\prime} Z}^{\prime}(x)=$ $-\frac{x^{2}}{1+x^{2}}$.

(123) Suppose $Z \subseteq]-1,1[$. Then

(i) (the function exp) (the function arctan) is differentiable on $Z$, and

(ii) for every $x$ such that $x \in Z$ holds ((the function exp) (the function $\arctan ))_{\uparrow Z}^{\prime}(x)=\exp x \cdot \arctan x+\frac{\exp x}{1+x^{2}}$.

(124) Suppose $Z \subseteq]-1,1[$. Then

(i) (the function exp) (the function arccot) is differentiable on $Z$, and

(ii) for every $x$ such that $x \in Z$ holds ((the function exp) (the function $\operatorname{arccot}))_{\uparrow Z}^{\prime}(x)=\exp x \cdot \operatorname{arccot} x-\frac{\exp x}{1+x^{2}}$.

(125) Suppose $Z \subseteq \operatorname{dom}\left(\frac{1}{r}((\right.$ the function $\left.\arctan ) \cdot f)-\operatorname{id}_{Z}\right)$ and for every $x$ such that $x \in Z$ holds $f(x)=r \cdot x$ and $r \neq 0$ and $-1<f(x)<1$. Then

(i) $\frac{1}{r}(($ the function $\arctan ) \cdot f)-\operatorname{id}_{Z}$ is differentiable on $Z$, and 
(ii) for every $x$ such that $x \in Z$ holds $\left(\frac{1}{r}((\right.$ the function $\arctan ) \cdot f)-$ $\left.\operatorname{id}_{Z}\right)_{\uparrow Z}^{\prime}(x)=-\frac{(r \cdot x)^{2}}{1+(r \cdot x)^{2}}$

(126) Suppose $Z \subseteq \operatorname{dom}\left(\left(-\frac{1}{r}\right)((\right.$ the function arccot $\left.) \cdot f)-\operatorname{id}_{Z}\right)$ and for every $x$ such that $x \in Z$ holds $f(x)=r \cdot x$ and $r \neq 0$ and $-1<f(x)<1$. Then

(i) $\left(-\frac{1}{r}\right)(($ the function $\operatorname{arccot}) \cdot f)-\operatorname{id}_{Z}$ is differentiable on $Z$, and

(ii) for every $x$ such that $x \in Z$ holds $\left(\left(-\frac{1}{r}\right)((\right.$ the function arccot $) \cdot f)-$ $\left.\operatorname{id}_{Z}\right)^{\prime}{ }_{\lceil Z}(x)=-\frac{(r \cdot x)^{2}}{1+(r \cdot x)^{2}}$.

(127) Suppose $Z \subseteq \operatorname{dom}(($ the function $\ln$ ) (the function arctan)) and $Z \subseteq$ ]$-1,1[$. Then

(i) (the function $\ln$ ) (the function arctan) is differentiable on $Z$, and

(ii) for every $x$ such that $x \in Z$ holds ((the function $\ln$ ) (the function $\arctan ))_{\uparrow Z}^{\prime}(x)=\frac{\arctan x}{x}+\frac{\text { (the function } \ln )(x)}{1+x^{2}}$.

(128) Suppose $Z \subseteq \operatorname{dom}(($ the function $\ln$ ) (the function arccot)) and $Z \subseteq$ ]$-1,1[$. Then

(i) (the function $\ln$ ) (the function arccot) is differentiable on $Z$, and

(ii) for every $x$ such that $x \in Z$ holds ((the function ln) (the function $\operatorname{arccot}))^{\prime}{ }^{\prime}(x)=\frac{\operatorname{arccot} x}{x}-\frac{(\text { the function ln) }(x)}{1+x^{2}}$.

(129) Suppose $Z \subseteq \operatorname{dom}\left(\frac{1}{f}\right.$ the function $\left.\arctan \right)$ and $\left.Z \subseteq\right]-1,1[$ and for every $x$ such that $x \in Z$ holds $f(x)=x$. Then

(i) $\frac{1}{f}$ the function arctan is differentiable on $Z$, and

(ii) for every $x$ such that $x \in Z$ holds $\left(\frac{1}{f}\right.$ the function $\arctan ^{\prime}{ }_{\mid Z}(x)=$ $-\frac{\arctan x}{x^{2}}+\frac{1}{x \cdot\left(1+x^{2}\right)}$.

(130) Suppose $Z \subseteq \operatorname{dom}\left(\frac{1}{f}\right.$ the function arccot) and $\left.Z \subseteq\right]-1,1[$ and for every $x$ such that $x \in Z$ holds $f(x)=x$. Then

(i) $\frac{1}{f}$ the function arccot is differentiable on $Z$, and

(ii) for every $x$ such that $x \in Z$ holds $\left(\frac{1}{f}\right.$ the function $\operatorname{arccot}^{\prime}{ }_{i Z}(x)=$ $-\frac{\operatorname{arccot} x}{x^{2}}-\frac{1}{x \cdot\left(1+x^{2}\right)}$.

\section{REFERENCES}

[1] Grzegorz Bancerek. The ordinal numbers. Formalized Mathematics, 1(1):91-96, 1990.

[2] Czesław Byliński. Functions and their basic properties. Formalized Mathematics, 1(1):5565, 1990.

[3] Czesław Byliński. Partial functions. Formalized Mathematics, 1(2):357-367, 1990.

[4] Pacharapokin Chanapat, Kanchun, and Hiroshi Yamazaki. Formulas and identities of trigonometric functions. Formalized Mathematics, 12(2):139-141, 2004.

[5] Krzysztof Hryniewiecki. Basic properties of real numbers. Formalized Mathematics, 1(1):35-40, 1990.

[6] Jarosław Kotowicz. Partial functions from a domain to a domain. Formalized Mathematics, 1(4):697-702, 1990.

[7] Jarosław Kotowicz. Partial functions from a domain to the set of real numbers. Formalized Mathematics, 1(4):703-709, 1990.

[8] Jarosław Kotowicz. Properties of real functions. Formalized Mathematics, 1(4):781-786, 1990. 
[9] Jarosław Kotowicz. Real sequences and basic operations on them. Formalized Mathematics, 1(2):269-272, 1990

[10] Konrad Raczkowski. Integer and rational exponents. Formalized Mathematics, 2(1):125$130,1991$.

[11] Konrad Raczkowski and Paweł Sadowski. Real function continuity. Formalized Mathematics, 1(4):787-791, 1990.

[12] Konrad Raczkowski and Paweł Sadowski. Real function differentiability. Formalized Mathematics, 1(4):797-801, 1990.

[13] Konrad Raczkowski and Paweł Sadowski. Topological properties of subsets in real numbers. Formalized Mathematics, 1(4):777-780, 1990.

[14] Yasunari Shidama. The Taylor expansions. Formalized Mathematics, 12(2):195-200, 2004.

[15] Andrzej Trybulec and Czesław Byliński. Some properties of real numbers. Formalized Mathematics, 1(3):445-449, 1990.

[16] Michał J. Trybulec. Integers. Formalized Mathematics, 1(3):501-505, 1990.

[17] Zinaida Trybulec. Properties of subsets. Formalized Mathematics, 1(1):67-71, 1990.

[18] Edmund Woronowicz. Relations defined on sets. Formalized Mathematics, 1(1):181-186, 1990.

[19] Yuguang Yang and Yasunari Shidama. Trigonometric functions and existence of circle ratio. Formalized Mathematics, 7(2):255-263, 1998.

Received March 18, 2008 